Proceedings

\title{
A Novel, Low-Cost, Portable PID Sensor for Detection of VOC ${ }^{+}$
}

\author{
Samuel O. Agbroko * and James Covington \\ University of Warwick, Coventry CV4 7AL, UK; j.a.covington@warwick.ac.uk \\ * Correspondence: o.s.agbroko@warwick.ac.uk; Tel.: +44-747-2408-854 \\ † Presented at the Eurosensors 2017 Conference, Paris, France, 3-6 September 2017. \\ Published: 7 August 2017
}

\begin{abstract}
A low cost portable photoionization (PID) sensor was successfully designed and manufactured. Unlike existing commercial PID sensors, our device provides two outputs, one associated with the total chemical components and a second that provides some level of compositional information. We believe that this makes this sensor system more useful than a standard PID, with a similar, if not lower, cost point. Our PID sensor was tested with gas concentrations down to $2 \mathrm{ppm}$ isobutylene. These results indicate that the limit of detection will be well below 1 ppm. Further detection tests were carried out with ethanol, acetone and isobutylene, which showed similar sensitivities. Compositional measurements were also undertaken and the results presented shows our sensor can discriminate successfully between low concentration isobutylene and 2-pentanone.
\end{abstract}

Keywords: PID; photo ionization; VOC detector

\section{Introduction}

Photoionization detectors (PID) are highly sensitive chemical sensors that are used extensively for the detection of volatile organic compounds (VOCs). They can provide a linear output to a single chemical or mix of chemicals, in real-time, and have a sensitivity in the ppb (parts per billion) range $[1,2]$. Their ability to analyze samples in ambient temperatures and pressures makes them suitable for testing in a range of environments and has resulted in their increased use for in situ analysis. Several PID sensors are commercially available from manufacturers including Alphasense and Mocon and cost approximately $\$ 500$ each. However, these sensors only provide a concentration value of the sample being tested, without any additional information of the chemical composition. Additionally, these sensors are not leak proof and cannot be easily used for inline analysis of toxic compounds.

In this paper, we report on a novel PID design that could potentially further reduce the costs in comparison with existing commercial PID sensors and offer additional compositional information about the sample under test.

\section{Experimental}

\subsection{Design Overview}

Our sensor system was manufactured as a single PCB solution. One side of the PCB are the detection electrodes, with the electonics to process the signal on the other side. An ionisation chamber and fluidics are fitted over the detectors. The sensor system measures $7 \mathrm{~cm} \times 5 \mathrm{~cm} \times 4 \mathrm{~cm}$ and weighs $200 \mathrm{~g}$. In use, the sample travels from the inlet to the ionisation chamber where ions are generated. A high electric field is then used to deflect the stream of ions serving as a filter for ions reaching the detector. A diagram of our system is shown in Figure 1. 


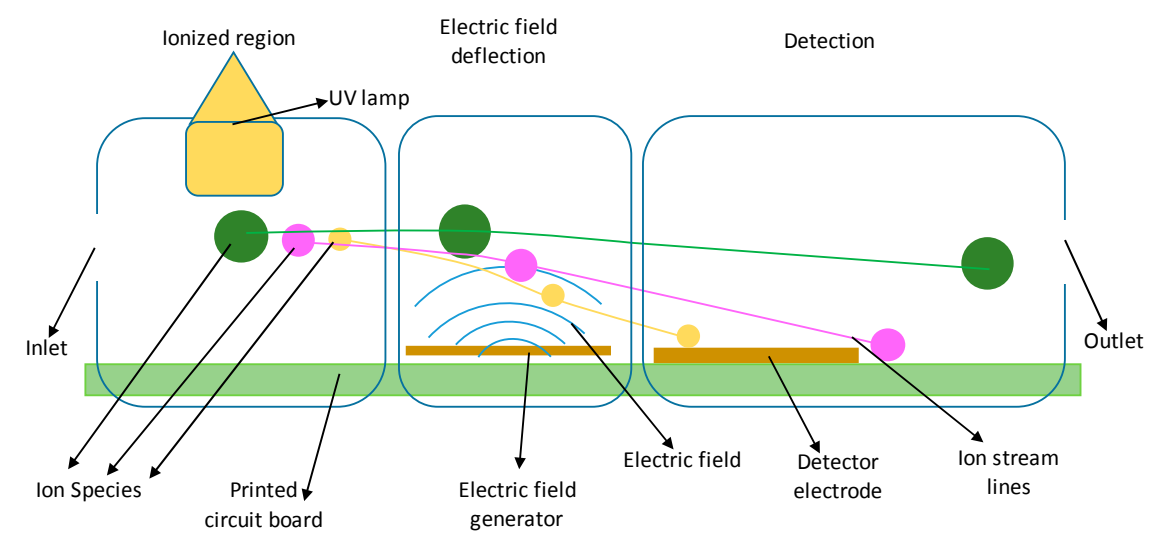

Figure 1. System illustration depicting the ionization, electric field and detection regions within the sensor.

\subsection{Ionisation}

The sample flows from the inlet to the ionisation chamber where it is ionised by high energy photons from a $10.6 \mathrm{eV} \mathrm{UV} \mathrm{lamp.} \mathrm{The} \mathrm{UV} \mathrm{lamp} \mathrm{is} \mathrm{mounted} \mathrm{on} \mathrm{the} \mathrm{ionisaiton} \mathrm{chamber} \mathrm{transverse} \mathrm{to}$ the flow of the sample. The ionisation chamber was manufactured from a single block of aluminium and measures $4.5 \mathrm{~cm} \times 2 \mathrm{~cm} \times 1 \mathrm{~cm}$ externally (Figure 2a). The ionisation chamber extends across the PCB and provides cover for the detection electrodes. Twelve M2 screws are used to attach the chamber to the PCB with a $1 \mathrm{~mm}$ o-ring compressed between both parts. A $6.1 \mathrm{~mm}$ window in the chamber wall allows high energy photons into the chamber. The cavity under the UV lamp is constricted to the size of the UV window to ensure that all of the sample travelling through the chamber is exposed to the UV lamp.

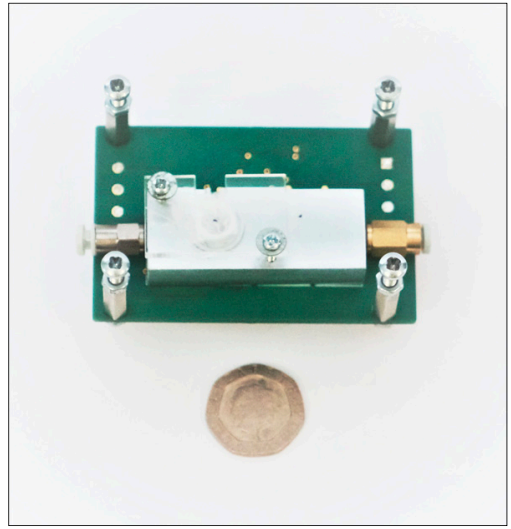

(a)

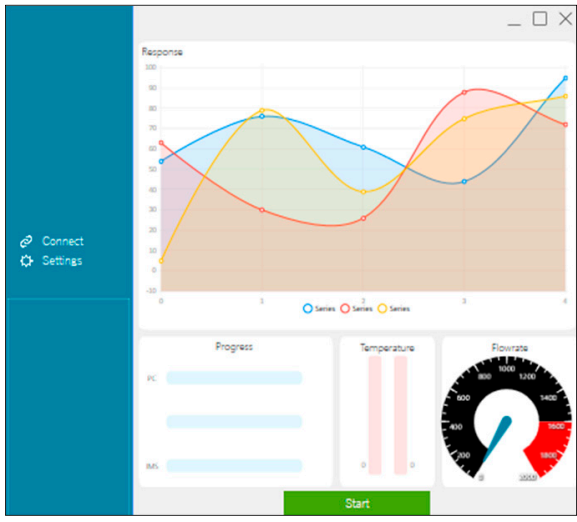

(b)

Figure 2. (a) Bottom view of the sensor showing the ionization chamber, UV lamp, inlet and outlet;

(b) The PID control software designed using Visual Studio 2017.

\subsection{Detection and Electronics}

The ionized sample enters the detection region, which comprises an of electric field region and a pair of detection electrodes. The electric field influences the motion of ions resulting in some selection of ions reaching the detectors. The electric field is held constant in one configuration. The output from this electrode is an indication of the total sample concentration (as with a traditional PID). The electric field for the second detector electrode is varied between $-7 \mathrm{kV} / \mathrm{m}$ and $7 \mathrm{kV} / \mathrm{m}$. This output provides some information on the composition of the sample. The detection electrodes, from both configurations, are connected to an amplification circuit that is implemented in two stages. In the first stage, a transimpedance amplifier is used to convert the detector current measured (in picoamperes) to a voltage. The second stage performs a basic amplification of the signal. A first order 
low pass filter is then used to remove noise before being read by a 12 bit ADC (analogue to digital converter). The device is controlled using an ATSAMD21G18 ARM Cortex M0 processor. This processor is interfaced with a computer via Bluetooth LE using the Nordic nRF51822 chipset. A C\# program was designed in-house to drive the electronics and record the amplified signals from the detection electrodes. The software also logs system parameters such as electric field, flowrate, concentration output and composition output over time.

\section{Results and Discussion}

\subsection{Concentration Information}

Isobutylene (ISB) is a common chemical used to characterize PID sensors. ISB was flowed into the device and its concentration was increased from $0-18 \mathrm{ppm}$ in steps of $2 \mathrm{ppm}$, by mixing the ISB with clean air. Figure 3 a shows the static voltage response with increasing isobutylene concentration, showing that the limit of detection is likely to be in the ppb region for ISB. To measure the dynamic characteristics, 10 ppm ISB was pulsed for $10 \mathrm{~s}$, followed by a $10 \mathrm{~s}$ rest, as shown in Figure $3 \mathrm{~b}$. The results indicate that the response time of our system is below $2 \mathrm{~s}$. Further testing was undertaken with ethanol, propanol and acetone at $50 \mathrm{ppm}$ concentrations. The results from these tests are shown in Figure 4.

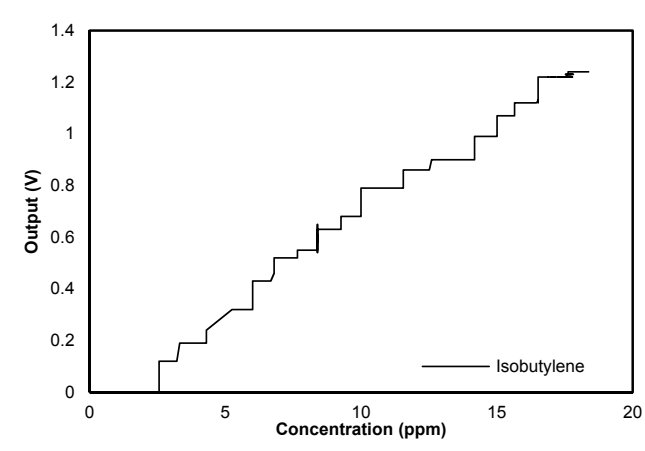

(a)

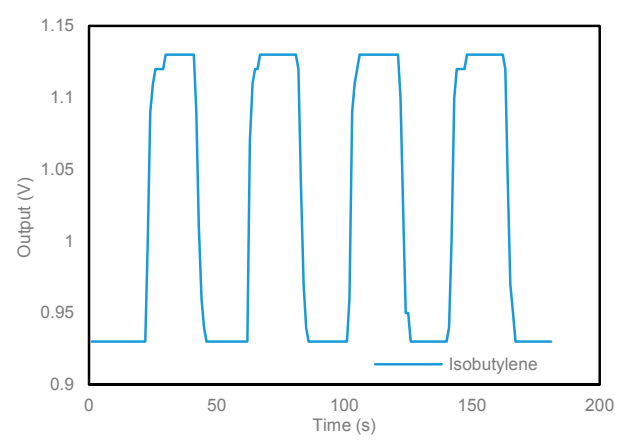

(b)

Figure 3. (a) Dynamic concentration response for isobutylene; (b) Dynamic response for 0 ppm and $15 \mathrm{ppm}$ isobutylene at $10 \mathrm{~s}$ intervals.

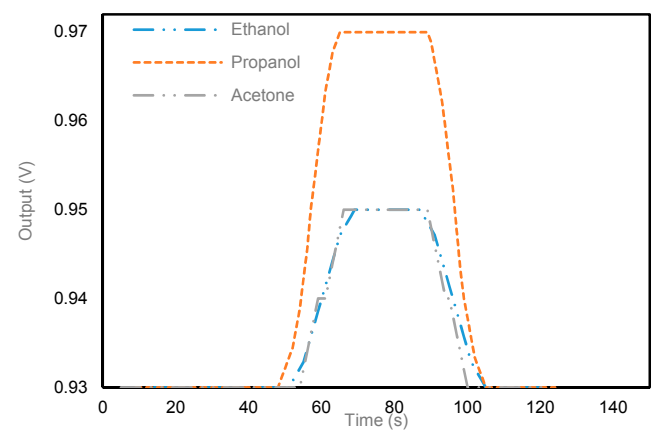

Figure 4. Concentration response for ethanol, propanol, acetone.

\subsection{Composition}

The second detector is used to provide some chemical composition information about the sample. To test this feature, the composition electrode was exposed to $10 \mathrm{ppm}$ ISB and $10 \mathrm{ppm} 2-$ pentanone. The test procedure involved supplying a pulsed flow of sample at $10 \mathrm{~s}$ intervals into the ionization chamber with the electric field swept from $-7 \mathrm{kV} / \mathrm{m}$ to $+7 \mathrm{kV} / \mathrm{m}$ in steps of $500 \mathrm{~V} / \mathrm{m}$. Figure 5 shows the output from the composition electrode for isobutylene and 2-pentanone. 


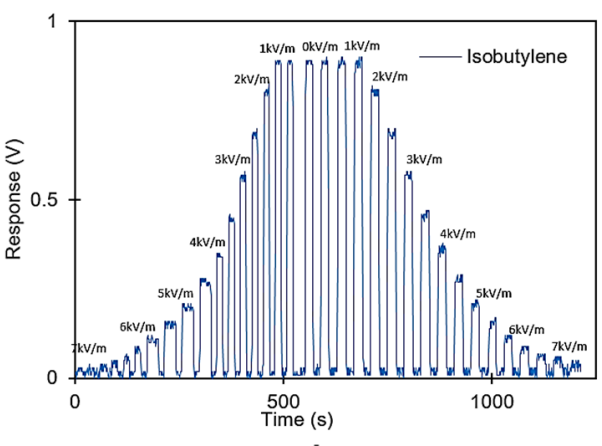

(a)

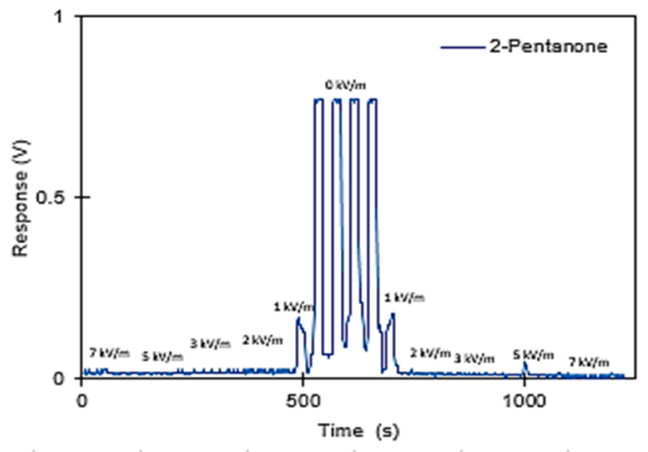

(b)

Figure 5. Composition electrode response with varying voltage in steps of $500 \mathrm{kV} / \mathrm{m}$ (a) isobutylene; (b) 2-pentanone.

Figure 5 illustrates that there are significant differences in the output voltage for isobutylene and 2-pentanone over the range of applied electric fields. Applying a $1 \mathrm{kV} / \mathrm{m}$ on the composition electrode results in a $0.67 \mathrm{~V}$ separation between the compounds.

\section{Conclusions}

In this paper, we report on a novel PID sensor with the addition of a sample composition output that has been designed, manufactured and tested. The sensor provides two voltage outputs proportional to the concentration and composition of the test sample. Concentration tests were carried out with isobutylene, ethanol, propanol and acetone. The concentration response was found to increase linearly with concentration for the chemicals under test. For isobutylene, an increase in concentration in from $0-18$ ppm resulted in an increase in output voltage of $2.3 \mathrm{~V}$, with an estimated sensitivity of below $1 \mathrm{ppm}$. The composition electrode response shows a significant distinction between 2-pentanone and isobutylene when $1 \mathrm{kV} / \mathrm{m}$ was applied in the chamber. The added composition information, leak proof feature and low cost makes this unit potentially provide more information at a lower price point when compared with existing commercial sensors.

Conflicts of Interest: The authors declare no conflict of interest.

\section{References}

1. Szulczyński, B.; Gębicki, J. Currently commercially available chemical sensors employed for detection of volatile organic compounds in outdoor and indoor air. Environments 2017, 4, 21, doi:10.3390/environments4010021.

2. Zhang, W.-Q.; Li, H.; Zhang, Y.-J.; Bi, F.; Meng, L.-S.; Zhang, X.-M.; Mao, J.-Y.; Cheng, N.-L.; Fang, B.; Yang, Y.; et al. Fast determination of monocyclic aromatic hydrocarbons in ambient air using a portable gas chromatography-photoionization detector. Chromatographia 2017, doi:10.1007/s10337-017-3331-1. 